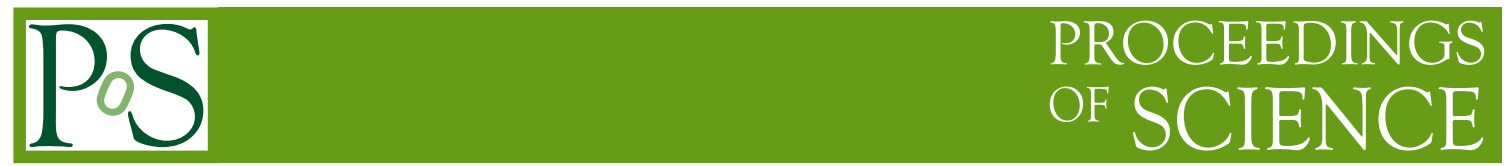

\title{
EFT results with top quarks in CMS
}

\section{Juan R. González Fernández on behalf of the CMS Collaboration}

\author{
University of Oviedo, Spain
}

E-mail: juan.rodrigo.gonzalez.fernandez@cern.ch

Recent results from CMS of searches for new physics in the context of effective field theories (EFT) are presented. A measurement of the $\bar{t} \gamma$ differential production cross section is performed and the results are interpreted in terms of EFT. A search for new physics through EFT in events with top quarks and multilepton final states is shown. A new EFT search using $t(t) Z$ events and machine learning techniques is presented as well. The three results are compatible with the standard model predictions and constraints in several Wilson coefficients are set.

The Ninth Annual Conference on Large Hadron Collider Physics - LHCP2021

7-12 June 2021

Online 


\section{Introduction}

The standard model (SM) of particle physics is one of the most accurate models to date. However, the SM cannot provide an explanation to the hierarchy problem, the existence of dark matter or other open questions. During the last decade, several searches for new physics have been performed by the experiments of the CERN Large Hadron Collider, but the results do not shed any light of new physics.

The effective field theory (EFT) formalism allows us to probe the existence of new physics at an energy scale $(\Lambda)$ above that of the LHC. The EFT lagrangian can be written as

$$
\mathcal{L}_{\mathrm{EFT}}=\mathcal{L}_{\mathrm{SM}}+\sum_{d, i} \frac{c_{i}^{d}}{\Lambda^{d-4}} O_{i}^{d},
$$

where $O_{i}^{d}$ are the EFT operators of dimension $d$, and $c_{i}^{d}$ are dimensionless parameters known as Wilson coefficients (WC) that characterize the strength of the interactions at dimension $d$.

In this document, three recent results by the CMS Collaboration [1] are presented. These results focus on dimension six operators, as higher dimensions are suppressed by additional powers of $\Lambda$.

\section{EFT results in $\bar{t} \bar{\gamma}$ events}

The measurement of the production cross section of a top quark pair in association with a photon is performed in events with an electron or muon and jets in the final state in CMS [2], using a data set corresponding to $137 \mathrm{fb}^{-1}$. The inclusive cross section is measured to be $800 \pm 46$ (syst) \pm 7 (stat) fb, in good agreement with the SM prediction.

The differential cross section is measured in several kinematic observables. The measurement as a function of the photon transverse momentum $\left(p_{\mathrm{T}}^{\gamma}\right)$, shown in Fig. 1 (left), is sensitive to the values of two WCs, $c_{t Z}$ and $c_{t Z}^{I}$, which corresponds to electroweak dipole moment operators described in [2]. An EFT interpretation is performed using Monte Carlo (MC) events at generation level, and a reweighing procedure is derived to modify the expected signal as a function of $\mathrm{c}_{\mathrm{tZ}}$ and $c_{\mathrm{tZ}}^{\mathrm{I}}$. Confidence intervals are set for the values of the two WCs, which are compatible with the SM expectation. The limits on $c_{\mathrm{tZ}}$ and $\mathrm{c}_{\mathrm{tZ}}^{\mathrm{I}}$, shown in Fig. 1 (right), are the most stringent to date.

\section{EFT analysis in multilepton final states}

A search for new physics through EFT in events with top quarks in multilepton final states is performed by CMS [3], using a data set corresponding to $41.5 \mathrm{fb}^{-1}$. Selected events must contain at least three leptons or two same-sign leptons and b-tagged jets. Signal events mostly come from associated top quark production. Background events come mainly from diboson production and nonprompt leptons.

A novel technique is used to parameterize the effect of $16 \mathrm{WC}$ on the predicted yields [3]. MC events are generated with different weights and different values for the WCs. A weight function is 

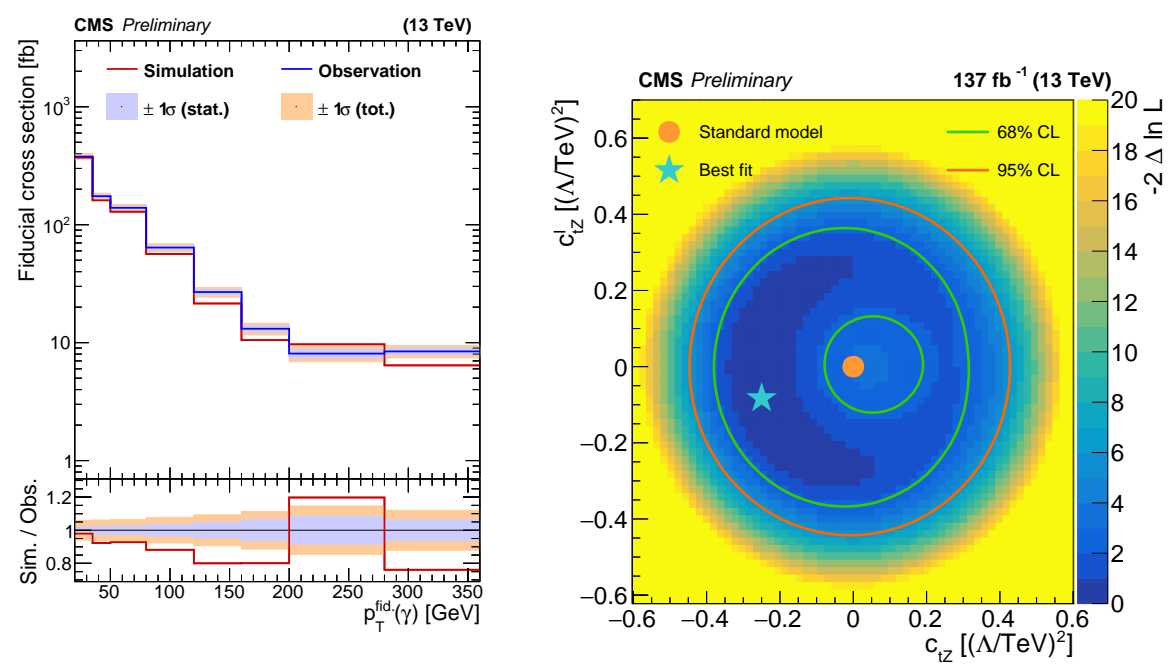

Figure 1: Differential cross section for $p_{\mathrm{T}}^{\gamma}$ compared with simulation from several predictions (left) and confidence intervals on $c_{\mathrm{tZ}}$ and $\mathrm{c}_{\mathrm{tZ}}^{\mathrm{I}}$ from a the two-dimensional scan of the two WCs (right). [2].

obtained for each event by performing a quadratic fit as

$$
w_{i}\left(\frac{\vec{c}}{\Lambda^{2}}\right)=s_{0 i}+\sum_{j} s_{1 i j} \frac{c_{j}}{\Lambda^{2}}+\sum_{j} s_{2 i j} \frac{c_{j}^{2}}{\Lambda^{4}}+\sum_{j, k} s_{3 i j k} \frac{c_{j}}{\Lambda^{2}} \frac{c_{k}}{\Lambda^{2}},
$$

where $\vec{c}$ are the WCs. The fit parameters are obtained for each event. A weight function for the expected yields in a given category is obtained by adding the weights of all the events in that category.

Events are divided into different categories depending on the number of leptons, flavour and charge of the leptons and number of b-tagged jets. To obtain confidence intervals for the EFT parameters, a simultaneous fit is performed to all the categories in which $16 \mathrm{WC}$ are profiled. The fit is repeated profiling each individual WC while keeping the rest at the SM value. The results are mostly compatible with the SM within $1 \sigma$ deviation.

\section{EFT results in $t(t) Z$ events}

A search for new interactions in the context of EFT is performed using associated top quark production with a $\mathrm{Z}$ boson in multilepton final states [4], using a data sample that corresponds to an integrated luminosity of $137 \mathrm{fb}^{-1}$.

Events containing at least 3 leptons, at least 2 jets, at least $1 \mathrm{~b}$-tagged jet and a $\mathrm{Z}$ boson candidate are selected. A neural network classifier is used to separate events into two signal categories (tZq, $\mathrm{t} \bar{Z}$ ) and a background category.

The effect of the EFT is parameterized using the same technique as in the previous analysis. $5 \mathrm{WCs}$ are considered and weight functions are obtained for each event using the equation (2). A set of binary neural network classifiers are trained to discriminate characteristic EFT events from $\mathrm{SM}$ events. Each classifier targets a specific WC for an individual signal process (tZq, tṫZ). Fig. 2 shows post-fit distributions for the output of two binary classifiers. 

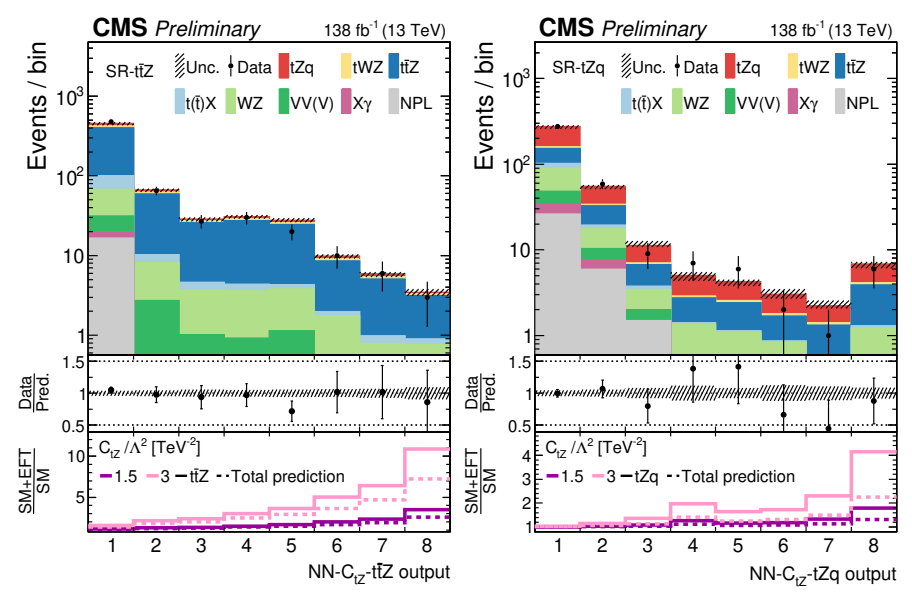

Figure 2: Post-fit data-to-simulation comparisons for the distributions used in the simultaneous fit to extract limits on $\mathrm{c}_{\mathrm{tZ}}$ for $\mathrm{t} \overline{\mathrm{t} Z}$ (left) and $\mathrm{tZq}$ (right) signal regions. The middle panels display the ratios of the observed event yields to their predicted values. The lower panel shows the change of the event yield in each bin with respect to the SM prediction for two arbitrary EFT scenarios. [4].

Results are extracted from a simultaneous fit to the data in six event categories that include different control regions, signal regions and the output of the binary classifiers. Confidence intervals are obtained with a simultaneous fit for the 5 explored WC (5D fit) and with individual fits for each WC where other WCs are set to the SM value. The results are shown in Table 1.

\begin{tabular}{|c|c|c|c|c|}
\hline \multirow[b]{3}{*}{$\mathrm{W}$} & \multicolumn{2}{|r|}{ Other WCs fixed to SM } & \multicolumn{2}{|c|}{ 5D fit } \\
\hline & Expected & Observed & Expected & Observed \\
\hline & \multicolumn{4}{|c|}{ 95\% CL confidence intervals } \\
\hline $\mathrm{c}_{\mathrm{tZ}}$ & {$[-0.97,0.96]$} & {$[-0.76,0.71]$} & {$[-1.24,1.17]$} & {$[-0.85,0.76]$} \\
\hline$c_{\mathrm{tW}}$ & {$[-0.76,0.74]$} & {$[-0.52,0.52]$} & {$[-0.96,0.93]$} & {$[-0.69,0.70]$} \\
\hline$c_{\varphi \mathrm{Q}}^{3}$ & {$[-1.39,1.25]$} & {$[-1.10,1.41]$} & {$[-1.91,1.36]$} & {$[-1.26,1.43]$} \\
\hline$c_{\varphi \mathrm{Q}}^{-}$ & {$[-2.86,2.33]$} & {$[-3.00,2.29]$} & {$[-6.06,14.09]$} & {$[-7.09,14.76]$} \\
\hline$c_{\varphi t}$ & {$[-3.70,3.71]$} & {$[-21.65,-14.61] \cup[-2.06,2.69]$} & {$[-16.18,10.46]$} & {$[-19.15,10.34]$} \\
\hline
\end{tabular}

Table 1: Expected and observed 95\% CL confidence intervals for all WCs. The intervals in the first and second columns are obtained by scanning over a single WC, while fixing the other WCs to their SM values of zero.

\section{Conclusions}

Three recent results of effective field theory interpretations with top quarks by CMS have been presented. A differential cross section of the $\bar{t} \gamma$ process is interpreted in terms of EFT to set limits on the value of two WCs. A search for new physics is performed using events containing top quarks in multilepton final states, in which 16 WCs are simultaneously fit. Finally, a EFT analysis using $\mathrm{tZq} / \mathrm{t} \mathrm{tZ}$ events and novel machine learning techniques has been presented. All the results, presented as confidence intervals on different WC, are compatible with the SM prediction. 


\section{References}

[1] CMS Collaboration, "The CMS experiment at the CERN LHC, JINST 3 (2008) S08004", doi:10.1088/1748-0221/3/08/S08004.

[2] CMS Collaboration, "Measurement of the inclusive and differential $\bar{t} \gamma$ cross sections in the single-lepton channel and EFT interpretation at $\sqrt{s}=13 \mathrm{TeV}$ ", submitted to JHEP, arXiv:2107.01508.

[3] CMS Collaboration, "Search for new physics in top quark production with additional leptons in proton-proton collisions at $\sqrt{s}=13 \mathrm{TeV}$ using effective field theory", JHEP 03 (2021) 095, doi: 10.1007/JHEP03(2021)095, arXiv:2012.04120.

[4] CMS Collaboration, "Probing effective field theory operators in the associated production of top quarks with a $\mathrm{Z}$ boson in multilepton final states at $\sqrt{s}=13 \mathrm{TeV}$ ", CMS-PAS-TOP-21-001, cds.cern.ch/record/2771677. 